\title{
Self-reproducing Automata and Polymetric Analysis
}

\author{
Petro Trokhimchuck \\ Dept. Theor. @ Math. Physics, Lesya Ukrayinka Eastern European National University, \\ Lutsk, Ukraine, 43025 \\ e-mail: trope1650@gmail.com
}

\begin{abstract}
Von Neumann concept of self-reproducing automats is discussed. Possible ways of resolution this problem is represented with point of Polymetric Analysis. Was shown that this problem may be resolved in general case as theory of functional-logical automata.
\end{abstract}

Index Terms - von Neumann; self-reproducing automata; Polymetrical Analysis; functional-logical automata; information calculations.

\section{INTRODUCTION}

The problem creation multifunctional theory of automata is basic problems of modern computer science [1-5]. We haave some ways of resolution this problem [3]. These are McCulloch-Pitts neural networks, Gödel's numbers, Kalman's polylinear machines, Fibonacci computers, systolic computers, neurocomputers [3], neural and cellular networks [3], a tensor analysis of the Cron contours and its diacoptic [3], quantum mechanical computers [3], fifth-generation computers, and most of all - self-reproducing von Neumann automata [1-3].

We already mentioned Gödel's numbers earlier [3]. No computers, including Fibonacci, quantum-mechanical, and fifth-generation computers have gone up to Gödel's numbers. For the first time, Gödel's numbers are very well used for theoretical analysis of computers and for the creation of new types of computers, pointing out von Neumann $[1,2]$, and in more detail it was painted by M. Minsky [4]. In general, Gödel's numbers are the following values:

$$
N_{G}=A^{\alpha} B^{\beta} C^{\gamma} \text {, }
$$

Where $N_{G}, A, B, C, \alpha, \beta, \gamma \quad-$ numbers. With this representation of numbers it is easy to move from a numerical representation to a linguistic one. Let's say $A, B, C-\mathrm{a}$ numerical representation, $\alpha, \beta, \gamma$ - representation for letters, and (1) - linguistic. But here comes the question, which is one of the main issues of modern cybernetics: how to link the cybernetics itself (analysis) with the theory of discrete automata, including the Gödel numbers. Most successfully it is connected in living automata, which, for example, is a man.

Let's dwell on von Neumann's self-reproducing automatic devices $[1,2]$. The theory of automata is a science of the basic principles common to artificial machines (digital computers, analogous computers, control systems) and natural automata (human nervous system, self-reproducing cells, organisms in the evolutionary aspect).
Von Neumann idea of reproducing may be connected with concept of Polymetric Analysis. Results of this synthesis is theory of functional-logical automata [3].

\section{RESULTS AND DISCUSSIONS}

Von Neumann set himself the problem of creation a system theory that would regulate the concepts and principles relating to the nature and organization of natural and artificial systems, the role of language and information in such systems, programming and management of such systems. It should be noted that from the very beginning the idea of feedback was established in cybernetics, starting with McCulloch-Pitts and Wiener [2, 3]. Von Neumann's concept is not present, but it's always used.

Let us now turn to self-reproduce in any natural and artificial systems. First, consider the idealized synchronous digital computing machine, which is constructed only from switches (eg "YES", "OR" and "NO") and delay lines (which delay pulses per unit time). Such a computer is a finite automaton having a finite number of parts and a finite number of states. Let the knot of the tape be an input, which, having received the appropriate command from the computer machine, mixes its tape on the cell to the front and that can read the contents of the viewed cell for the computer. Let's have a similar output, let there be another knot of the tape that is capable of recording in the cell of its tape and move this tape forward, receiving a corresponding signal from the computer.

Thus, we have a finite automaton with incoming and outgoing knots of the tape. Such a machine can be converted into a Turing machine by attaching to it a third knot of the tape, which can move the tape in both directions, read from the tape and write to it. On the basis a finite automaton, which does not require much complexity, we have a universal computing machine capable of performing any computation that can be performed on a particular computing machine.

In the class of these idealized computers, the central one of them, that is, a finite automaton, was synthesized from parts, which can not be said about the input and output knots of the tape, as well as the knots of the tape, which are intended for memorization. Moreover, the external environment for this machine can not be filed. Almost automata of this kind are one of the options of the McCulloch-Pitts chains [2]. For them, von Neumann proposed a kinematic model of self-reproducing [3]. He considers the following types of basic elements of the machine: switching element; delay lines; an element capable of perceiving (for example, hearing or seeing); action element 
(for example, an artificial arm); an element that can separate other elements; an element that is capable of connecting objects and a rod that allows you to build a rigid framework for ensemble elements.

In principle, from all these elements you can build a machine that can not only calculate, but also take, act, move, separate objects from each other and construct other machines. The external environment for these idealized kinematic automata can be imagined in the form of an unlimited ocean, on the surface of which an unlimited number of specimens of the main elements of each type floats. In this machine the switching elements, the sensory organs and the action organs dividing the organs that unite the organs and the rods, all of them carry out random motion, as gas molecules. Thus, each machine, which is placed on the surface of this ocean, is supplied by parts in unlimited quantities.

The entire process of kinematic self-reproducing is carried out as follows [3]. Imagine that on the surface of our ocean floats the kinematic machine, which is a finite automaton $M_{U}$ that is connected to the tape $T$. The basis of this tape is a zigzag chain from the rods, and in each section one more rod can be placed, symbolizing 1 , the absence of such a rod symbolizes 0 (Fig. 1) [3].

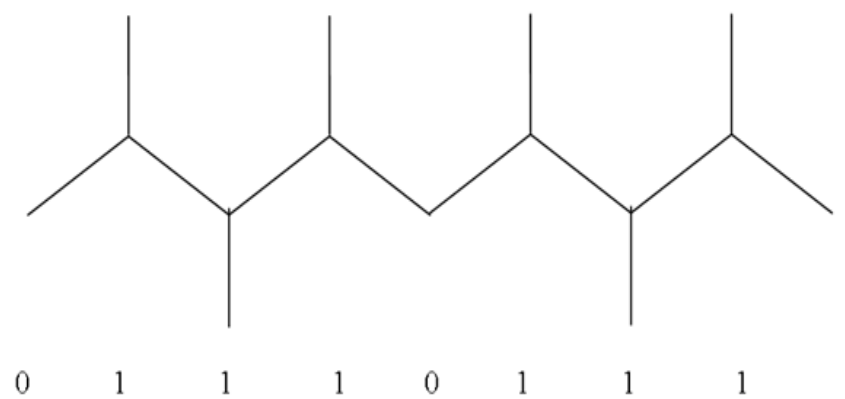

Fig. 1. A scheme for explaining the process of kinematic selfreproducing [3].

The finite automaton $M_{U}$ has the properties of a finite part of the universal computer - it can control the tape $T$ and interpret the arbitrary computational program that is on this tape. In addition, the machine $M_{U}$ has sensory, active, interconnecting and dividing bodies that are interconnected so that it can pick up the ocean surface rods and use them to change the information stored on the tape and for unlimited continuation of the tape. Therefore, a complex consisting of a machine $M_{U}$ and a tape $T$ is a universal computer.

On a set of kinematic automata, a universal machinedesigner generalizes a universal computing machine. Any finite machine consists of a finite number of basic elements, which are connected in a certain way, and therefore it can be fully characterized by a finite description $D(M)$ that can be applied to the tape. Let $M_{C}$ it be a finite part of the universal designer machine. The designer $M_{C}$ has the capabilities of three types. Firstly, he can do all that a universal computer $M_{i j}$ can do. Secondly, he can construct any finite machine $M_{i j}$ when it is given a full description $M_{C}$ of it. To perform such a design, $M_{C}$ reads the description $D(M)$, finds and collects the parts that are needed for it, and connects them to each other in accordance with the plan $D(M)$. Thirdly, he can copy on a new tape and attach the tape to $M$.

Let the knot, which we want to construct, consist of an arbitrary machine $M$ and an attached tape $D(M)$ to it. A universal designer, provided that the ribbon contains a description $D(M)$, acts like this. Firstly $M_{C}$ reads $D(M)$, takes the necessary parts for $M$ and connects them together in accordance with the plan $D(M)$. Thus, a universal designer, drawing on the description and having the corresponding entry of parts, constructs a machine with a ribbon $D(M)$. The general scheme of this construction is as follows:

1) $M_{C}$ with a ribbon $D(M)$ desidn $M$ with a ribbon $D(M)$.

Note that the machine that is being constructed contains its own description. To get self-reproducing as a partial case of this general scheme, we replace $M$ in point 1 by and then we obtain:

2) $M_{C}$ from ribbon $D(M)$ constructs $M_{C}$ with tape $D(M)$.

When performing part 2 a universal designer $M_{C}$ first describes $D\left(M_{C}\right)$, selects the parts that are needed for $M_{C}$, and connects them in accordance with the plan $D\left(M_{C}\right)$. Then he makes a copy of the tape $D\left(M_{C}\right)$ and attaches it to $M_{C}$. A universal designer does all this, not knowing what his copy is being made and copying his description. However, the entire process starts with one copy $M_{C}$ with a ribbon $D(M)$ and ends with two copies of this complex. This is a self-reproducing. The scheme of described self- reproducing is shown in Fig. 2 [3].

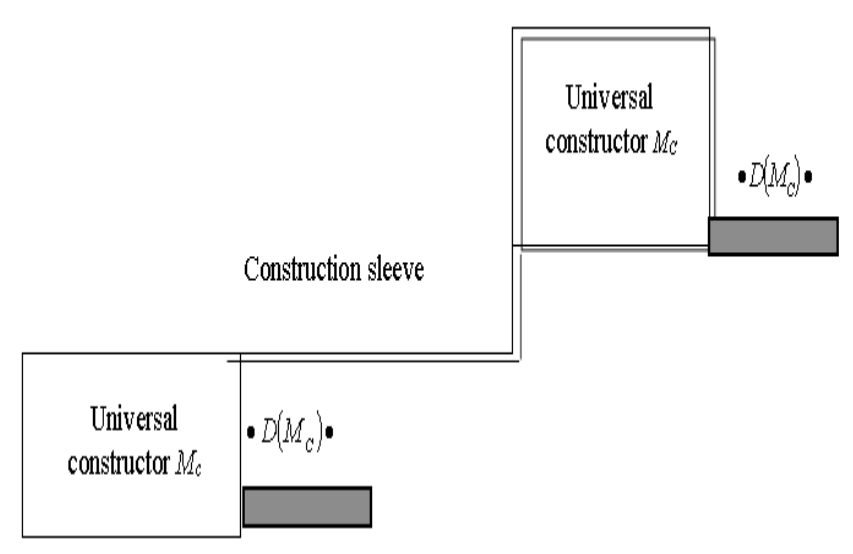

Fig. 2. Scheme of kinematic self-reproducing [2]. 
Block diagram description of self-reproducing here is presented in general terms. But even with such an outline there is an analogy between self-reproducing of automatic machines and the process of self- reproducing of living cells. The tape $T$ with the description $D(M)$ is similar to the chain of deoxyribonucleic acid (DNA) carrying genetic information in the nucleus of the cell. The copying operation of the tape $D(M)$ is similar to DNA replication, which is carried out under the control of the DNA polymerase enzyme. Finally, the design of an automaton $M$ on the basis $D(M)$ is a similar construction of a new cell.

However, the kinematic model of self-reproducing automata is rather complicated. Firstly, the kinematic characteristics of the model are far removed from chemistry, physics and mechanics, in order to have more interest by themselves, and from another point of view, they are very far from the problems of organization management and logic, in order to increase our understanding of these problems. Thus, the motor capabilities of the kinematic system are only a source of complexity, which in our context do not justify the cost, and therefore it is expedient to exclude them.

The cellular automaton eliminates these complexities. It consists of an unlimitedly integrated, or mosaic, array of finite automata, each of which interacts with its neighbors. It depicts in this way the main "frames" or "space" in which there are events related to the operation of the machine and for which we can formulate precise and simple rules or laws.

There are many types of cellular automata [3, 4]. You can specify the geometry of the cell space, the neighborhood relation, the types of automata, the laws of transitions for the system, and the initial state of the system. Von Neumann uses a two-dimensional array ("chess board"), in which each finite state machine has 29 states.

From the main result of this approach, it follows that on this system, cells with 29 states can be designed universal machines designers and self-reproducing machines.

In the case of self- reproducing, the finite number of cells from the very beginning is organized in such a way as to form a universal automaton constructor $M_{C}$. His description $D\left(M_{C}\right)$ appears in a finite linear array of cells that act as a ribbon, while the other part of the cellular system (environment) remains in a passive, unorganized state. When the machine $M_{C}$ is activated, it works both as a computer and as a constructor, sending information in the cellular space that makes it possible to organize a copy of the machine $M_{C}$ and the tape $D\left(M_{C}\right)$ in another finite part of the space. Thus, the initial finite organization of this system is self-reproducing.
Consequently, in comparison with the kinematic scheme, the cellular pattern of self- reproducing automaton is informationally superplus. But von Neumann believed that all the operations of cellular automata, as well as the McCullochPitts net, could be reduced to logical operations. However, the very idea of information superplus, strictly speaking, does not coincide very well with the classical logic. It is suitable for polylinear structures, that is, structures that can be reduced to one level of the hierarchy, if expressed in the language of the theory of systems. The von Neumann self- reproducing devices belong to these and belong, since they restore similar ones to themselves, although cellular machines already have some kind of difficulty connected with polynomiality. Von Neumann wanted to create automata [3] that could solve differential equations. But in mathematics there is Sato's theorem [234], which shows that almost all of the differential nonlinear equations can be reduced to systems with a greater number of linear differential and ordinary algebraic equations that have the same solutions as the desired nonlinear differential equations. The question arises whether you need to create computers that would solve the problems of classical mathematics. Is it not better to develop a mathematical analysis that would be most effective for computers but not for the classics, but with the same clarity as the classics. At the end of the review background von Neumann works, which, unfortunately, have not been completed, we present another scheme of self- reproducing automatic machine (Fig.3.9), which has a direct relation to the appearance of functional logic $[3,4]$, which, as we emphasized above, is a synthesis of mathematical logic and logistics, therefore that such a synthesis makes it easier to describe the automata of the type, which is shown in Fig. 3 [3].

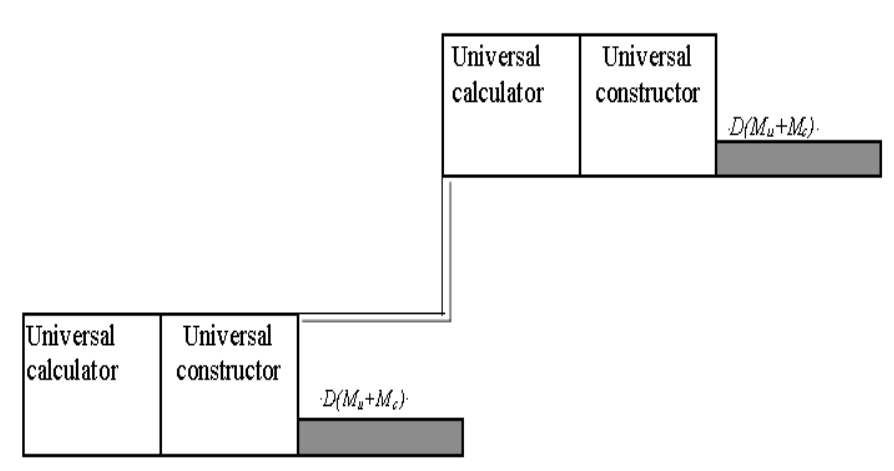

Fig. 3. The scheme of cellular von Neumann automata [2].

Now we go to concept of functional-logical automata according to [4]. We must represent basic concepts of Polymetric Analysis [4].

Definition 1. The mathematical constructive elements $N_{\varphi_{i j}}$ are called the functional numbers

$$
N_{\varphi_{i j}}=\varphi_{i} \circ \bar{\varphi}_{j} .
$$


Where $\varphi_{i}\left(x_{1}, \ldots x_{n}, \overline{x_{1}} \ldots \bar{x}_{m}, \ldots N_{x_{i j}}, \ldots\right)$,

$\overline{\varphi_{j}}\left(x_{1}, \ldots x_{n}, \overline{x_{1}} \ldots \bar{x}_{m}, \ldots N_{x_{i j}}, \ldots\right)$ are the straight and opposite functions, respectively; $\circ$ - respective mathematical operation.

Basic elements of PA is the generalizing mathematical elements or its various presentations - informative knots [4]. Generalizing mathematical element is the composition of functional numbers (generalizing quadratic forms, including complex numbers and functions) and generalizing mathematical transformations, which are acted on these functional numbers in whole or its elements [1]. Roughly speaking these elements are elements of functional matrixes.

This element ${ }_{n \text { mab }}^{\text {sto }} M_{i j k p}$ may be represented in next form

$$
{ }_{n m a b}^{s t q o} M_{i j k p}=A_{i} \bar{A}_{j} O_{k} \bar{O}_{p} A_{s}^{r} \bar{A}_{t}^{r} O_{q}^{r} \bar{O}_{o}^{r} A_{n}^{l} \bar{A}_{m}^{l} O_{a}^{l} \bar{O}_{b}^{l} N_{\varphi_{i j}} \text {. }
$$

Where $\quad N_{\varphi_{i j}} \quad-\quad$ functional number; $O_{k}, O_{q}^{r}, O_{a}^{l}, \bar{O}_{p}, \bar{O}_{o}^{r}, \bar{O}_{b}^{l} ; A_{i}, A_{s}^{r}, A_{n}^{l}, \bar{A}_{j}, \bar{A}_{t}^{r}, \bar{A}_{m}^{l}$ are quantitative and qualitative transformations, straight and inverse (with tilde), (r) - right and (l) - left. We have only 15 minimal types of generalizing mathematical transformations [4].

The set of this elements is created the informative lattice [4]. Theory of information calculations is based on informative lattice. Basic principle of this theory is principle of optimal informative calculations [4].

For classification of systems of calculation hybrid theory of systems was created. This theory is based on two criterions: criterion of reciprocity - principle of creation of proper formal system, and criterion of simplicity - principle of optimality of this creation. For "inner" bond of two elements of informative lattice a parameter of connectedness $\sigma_{t}$ was introduced. Principle of optimal informative calculation is included in criterion of simplicity. At help these criteria of reciprocity and simplicity and parameter of connectedness the basic famous parts of knowledge and science may be represent as 10 types of hybrid systems [3].

Definition 2 [4]. The functional-logical (polymetric) automaton is called the information lattice, which is constructed using a hybrid system approach, which provides super-modular operations and which can perform certain operations without human intervention.

The connections of this polyfunctional axpansion with other concepts of computer machine (automata) may represented as next theorems [3].

Theorem 1. Turing machine is a partial case of a functional logic machine [3].

Proof. For the "black box" of the Turing machine, let's take a functional number. Then the states of the Turing machine will be determined by the relations

$$
O_{n} \overline{O_{m}} N_{\varphi_{i j}} .
$$

In this case, if the rank of a zero-algebra is equal to infinity, then we have an infinite tape of the Turing machine; if the rank of zero-algebra is finite, then, respectively, the finite tape of the Turing machine.

Theorem 2. The von Neumann self-reproducing automata are a partial case of a functional-logical automaton.

Proof. If we specify in a certain way algebras $O_{n}$ and $\overline{O_{m}}$, then for each input $\left(O_{n}, \overline{O_{m}}\right)$ one output $O_{n} \bar{O}_{m} N_{\varphi_{i j}}$ will correspond.

In conclusion, we give a functional diagram of the possible implementation of a functional-logical computer (Fig. 4).

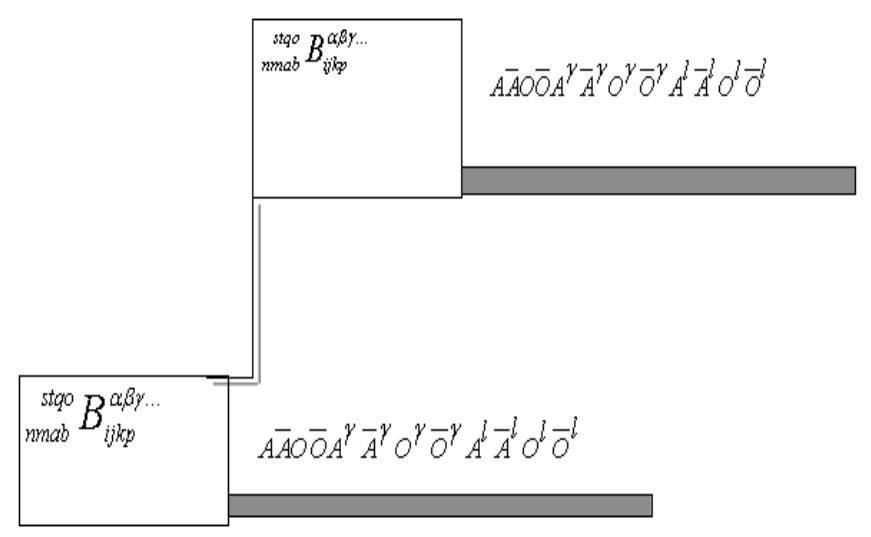

Fig. 4. Functional-logical scheme of self-reproducing [3].

In polymetric method the procedure of self-reproducing is included in informative knots and unite the universal calculator (quantitative transformations) and universal constructor (qualitative transformations) in one element [3].

\section{CONCLUSIONS}

1. Von Neumann concept of self-reproducing automata is analysed.

2. Basis knotions of polymetric analysis is represented.

3. Was shown, that theory of functional-logical automata may be realization of the resolution von Neumann problem.

\section{REFERENCES}

[1] J. Von Neumann , "The general and logical theory of automata.," In: A. M. Turing. "Can the machine think," Moscow: Fizmatgiz, 1960, pp..91192. (In Russian)

[2] J. Von Neumann, "The theory of self-reproducing automata," Moscow: Mir, 1971, 384 p. (In Russian)

[3] P. Trokhimchuck, "Polymetrical Analysis. History, Concepts, Applications," Saarbrukken: Lambert Academic Publishing, 2018, $280 \mathrm{p}$.

[4] M. Minsky, "Calculations and automata," Moscow: Mir, 1971, 375 p. (In Russian)

[5] A. I. Kuhtenko, "Cybernetics and Fundamental Science," Kyiv: Naukova Dumka, 1987, 144 p. (In Russian) 